\title{
Standard Blood Laboratory Values As A Clinical Support Tool to Distinguish Between SARS-CoV-2 Positive and Negative Patients
}

\author{
Rainer Thell \\ Department of Internal Medicine 2, Emergency Department, Klinik Donaustadt, 122 Langobardenstrasse, 1210 Vienna \\ Jascha Zimmermann \\ Sigmund Freud University, Medical School, Vienna \\ Marton Szell \\ Department of Internal Medicine 2, Emergency Department, Klinik Donaustadt, 122 Langobardenstrasse, 1210 Vienna \\ Sabine Tomez \\ Wiener Gesundheitsverbund, Vienna \\ Philip Eisenburger \\ Wiener Gesundheitsverbund, Vienna \\ Moritz Haugk \\ Wiener Gesundheitsverbund, Vienna \\ Anna Kreil \\ Wiener Gesundheitsverbund, Vienna \\ Alexander Spiel \\ Wiener Gesundheitsverbund, Vienna \\ Amelie Blaschke \\ Department of Internal Medicine 2, Emergency Department, Klinik Donaustadt, 122 Langobardenstrasse, 1210 Vienna \\ Anna Klicpera \\ Department of Internal Medicine 2, Emergency Department, Klinik Donaustadt, 122 Langobardenstrasse, 1210 Vienna \\ Oskar Janata \\ Department of Internal Medicine 2, Emergency Department, Klinik Donaustadt, 122 Langobardenstrasse, 1210 Vienna \\ Walter Krugluger \\ Department of Internal Medicine 2, Emergency Department, Klinik Donaustadt, 122 Langobardenstrasse, 1210 Vienna \\ Christian Sebesta \\ Department of Internal Medicine 2, Emergency Department, Klinik Donaustadt, 122 Langobardenstrasse, 1210 Vienna \\ Harald Herkner \\ Medical University Vienna \\ Brenda Laky ( $\square$ brenda.laky@meduniwien.ac.at) \\ Austrian Research Group for Regenerative and Orthopedic Medicine (AURROM), Hartmanngasse 15/10, 1050 Vienna
}

\section{Research Article}

Keywords: COVID-19, SARS-CoV-2, PCR, standard blood laboratory, eosinopenia

Posted Date: December 3rd, 2020

DOI: https://doi.org/10.21203/rs.3.rs-112467/v1

License: (c) (1) This work is licensed under a Creative Commons Attribution 4.0 International License. Read Full License 


\section{Abstract}

Standard blood laboratory parameters may have diagnostic potential, if polymerase-chain-reaction (PCR) tests are not available on time. We evaluated standard blood laboratory parameters of 655 COVID-19 patients suspected to be infected with SARS-CoV-2, who underwent PCR testing in one of five hospitals in Vienna, Austria. We compared laboratory parameters, clinical characteristics, and outcomes between positive and negative PCR-tested patients and evaluated the ability of those parameters to distinguish between groups. Of the 590 patients (20-100years, 276 females and 314 males), 208 were PCR-positive. Positive compared to negative PCR-tested patients had significantly lower levels of leukocytes, neutrophils, basophils, eosinophils, lymphocytes, neutrophil-to-lymphocyte ratio, monocytes, and thrombocytes; while significantly higher levels were detected with erythrocytes, hemoglobin, hematocrit, C-reactive-protein, ferritin, activated-partial-thromboplastin-time, alanine-aminotransferase, aspartate-aminotransferase, lipase, creatine-kinase, and lactate-dehydrogenase. From all blood parameters, eosinophils, ferritin, leukocytes, and erythrocytes showed the highest ability to distinguish between COVID-19 positive and negative patients (area-under-curve: 72.3-79.4\%). Leukopenia, eosinopenia, elevated erythrocytes, and hemoglobin were among the strongest markers regarding accuracy, sensitivity, specificity, positive and negative predictive value, positive and negative likelihood ratio, and post-test probabilities. Our findings suggest that especially leukopenia, eosinopenia, as well as elevated erythrocytes, hemoglobin, and ferritin are helpful to distinguish between COVID-19 positive and negative tested patients.

\section{Introduction}

In December 2019, an uncommonly high incidence of pneumonia occurred in Wuhan province, China, caused by a previously unknown pathogen and showing an unusually high mortality rate, which showed to be a novel corona virus, SARS-CoV-2 (Severe Acute Respiratory Syndrome - Corona Virus 2). ${ }^{1}$ It causes coronavirus disease (COVID-19) which has reached pandemic levels resulting in significant morbidity and mortality affecting all inhabited areas of the world with large numbers of patients. Hence, the World Health Organisation (WHO) declared a worldwide pandemic in March 2020.

Diagnostic steps for this disease currently are epidemiological contact history, clinical impression, chest radiography, standard blood laboratory, and antigen detection by means of real-time fluorescence PCR. PCR is the gold standard test for detection of SARS-CoV-2 infection. ${ }^{2}$

It soon became apparent that the available test capacities for PCR testing were far from sufficient, and a feverish search for alternative and simpler detection methods began. To date, point-of-care PCR testing is still not available everywhere. Testing that takes a long time can make up for significant additional efforts of organisation of patient cohorts in hospitals.

The clinical appearance of the disease is broadly reported. ${ }^{3}$ Signs and symptoms may include fever and cough most commonly, dyspnoea, rarely diarrhoea, anosmia, or ageusia and others, at the beginning or during the course of the disease. ${ }^{4-6}$ Various publications indicated that COVID-19 positive patients showed typical laboratory patterns, ${ }^{7-9}$ although many of the earlier studies report on relatively small numbers of COVID-19 positive patients.

Zhang et al. included 95 cases with COVID-19-positive pneumonia. ${ }^{7}$ They found significantly higher numbers of D-Dimer, C-reactive protein (CRP), and procalcitonin in patients to be admitted to intensive care levels. In a retrospective report of 138 admitted patients, lymphopenia occurred in $70 \%$, a prolonged prothrombin time in $58 \%$, and an elevated lactate dehydrogenase (LDH) resulted in $40 \%$ of patients. ${ }^{10}$ In an Iranian cohort of 70 COVID-19 positive patients, Mardani et al. found significantly higher neutrophil count, CRP, LDH, aspartate aminotransferase (AST), alanine aminotransferase (ALT), and urea levels, as well as lowered white blood count and albumin levels. ${ }^{11}$ A further retrospective trial of 99 patients showed decreased lymphocyte counts in 35\%, decreased albumin in 98\%, increased LDH in 75\%, an increased interleukin-6 in 52\%, and raised CRP in $86 \%$ of patients were reported. ${ }^{3}$ Li et al. performed a trial with 989 patients, detecting a combination of eosinopenia and elevated CRP yielding a sensitivity of $67 \%$ and specificity of $78 \%$ for COVID-19. ${ }^{12}$ In an additional cohort of 458 patients from Guan et al., leukopenia, lymphopenia, eosinopenia, and an increased CRP were detected. ${ }^{4}$ In two trials, an increased NLR was described as an independent risk factor of mortality for COVID-19 patients. ${ }^{13,14}$

Standard blood laboratory data are potentially of both, diagnostic and prognostic value. ${ }^{15}$ On one hand, they can contribute to judge the pre-test probability of a COVID-19 diagnosis and thus, support the effective and efficacious organisational management of a patient in an emergency department. On the other hand, it is of potential value, if standard blood laboratory blood results can help to distinguish a potentially lifethreatening course of a disease from a less critical status.

Therefore, the overall aim of this study was to evaluate standard blood laboratory parameters and clinical characteristics in a large number of COVID-19 suspicious patients, who underwent PCR testing. The specific aim was to determine whether standard blood laboratory parameters are able to identify positive COVID-19 patients from a large COVID-19 suspected cohort.

\section{Methods}


Data were obtained from an electronical data base of the Vienna Health Care Association (Wiener Gesundheitsverbund), which stores all medical records of patients treated in its hospitals in Vienna, Austria. Data extraction was exclusively performed by authorised employees of the emergency department at the Klinik Donaustadt, Vienna. The study protocol was approved both by the Ethics Commission of the City of Vienna (EK 20-122-VK) and the Ethics Commission of Sigmund Freud University Vienna (161/2020). Due to the retrospective character of the study, it did not infringe on patient's privacy or health. Both Ethics Commissions approved a waiver for informed consent. This study was carried out in accordance with relevant guidelines and regulations.

\section{Data Collection}

Data were collected from female and male adult patients with suspected COVID-19 who underwent reverse transcriptase PCR testing via a nasopharyngeal swab performed at one of the five hospitals (Klinik Donaustadt, Klinik Floridsdorf, Klinik Hietzing, Klinik Landstrasse, and Klinik Ottakring) between February 27, 2020 and April 27, 2020. Based on PCR results, patients were divided into a COVID-19 positive or negative group. Patients without reported standard blood laboratory reports, medical history, or outcome documentation at day 28 after consultation were not included.

As standard blood laboratory testing was performed according to the clinical care needs of the patients, not all parameters were available for all patients. Routine blood tests generally included full blood count, blood chemistry, electrolytes, liver function parameters, renal and myocardial function parameters as well as coagulation markers and markers of inflammation.

Patients' gender (female/male), age at time of PCR testing (years), coexisting diseases and conditions (e.g. chronic diseases of the lung, liver, kidney; coronary artery disease, diabetes, and arterial hypertension); the clinical 28-day outcome including hospital admission and discharge as well as requirement of intensive care and ventilation, and death details were extracted from medical records.

\section{Statistical Analysis}

Descriptive statistics was used to describe the characteristics of patients. Data distribution was determined by visual inspection of the histograms and the Kolmogorov Smirnov tests. Normally distributed data were calculated as mean value with standard deviation (SD), otherwise as median and interquartile range (IQR). Continuous variables were compared between COVID-19 positive and negative patients with independent t-tests (parametric) or Mann-Whitney U-tests (non-parametric). Only those continuous parameters with significant differences were further evaluated. Area under the ROC curves (AUC) were determined in order to assess the ability of continuous blood parameters to distinguish between COVID-19 positive and negative patients.

Blood parameters were categorised according to normal reference ranges used in hospitals. However, since CRP ranges higher than normal (cut off: $0.5 \mathrm{mg} / \mathrm{dL}$ ) were detected in almost all patients (COVID-19 positive: $99.5 \%$ and negative: $98.2 \%$ ) and thus, useless to discriminate between COVID19 positive and negative patients, coordinates of the receiver operating characteristic (ROC) and the Youden index were used to determine a, sensitivity and specificity balanced, cut off value for CRP in our cohort. A cut-off for neutrophils-to-lymphocytes ratio (NLR) was also determined using coordinates of the ROC and the Youden index (sensitivity and specificity balanced). Chi-square or Fisher's exact tests were applied to describe the relationship between proportions of categorical variables. ). Only those categorical parameters with significant differences were further evaluated. Accuracy, sensitivity, specificity, positive predictive value (PPV), negative predictive value (NPV), positive likelihood ratio (LR+), and negative likelihood ratio (LR-) were calculated for each parameter. Bayes' theorem was used to determine blood parameters' post-test probabilities, which were calculated using pre-test probabilities and likelihood ratios. Statistical significance was set at a p-value of $<0.05$ (two-sided). All data were analysed with SPSS software (IMP Statistics Version 25; SPSS Inc, Chicago, IL).

\section{Results}

\section{Characteristics of COVID-19 Suspected Patients}

A total of 655 patients from the four hospitals underwent PCR testing between February 27, 2020 and April 27, 2020. 45 patients were not evaluated due to missing data. Another 17 patients who were tested within this time period with complete data sets were excluded since they were hospitalized more than one month before $(n=16)$ or more than seven days after $(n=1)$ PCR-testing. Patients $(n=3)$ with eosinophilia and acute malignant disease were excluded as well.

The median age of the 276 female (46.8\%) and 314 male (53.2\%) patients was 71 years (range, 20-100years; $60.7 \%$ of the patients were $\geq 65$ years of age at time of PCR testing). No comorbidities were recorded in 69 (33.2\%) and 84 (22.0\%) COVID-19 positive and negative tested patients, respectively. COVID-19 negative tested patients had significantly more comorbidities than COVID-19 positive tested patients (median (IQR): 1 (1-3) vs. 1 (0-2); $\mathrm{p}<0.001)$. The most common comorbidity was pre-existing arterial hypertension (58.1\%), followed by diabetes ( $25.4 \%)$, coronary heart disease (19.5\%), chronic lung disease (16.9\%), chronic kidney disease (15.8\%), malignant diseases (13.6\%), cerebrovascular accidents (7.5\%), chronic liver disease (4.2\%), and human immunodeficiency virus (HIV, $0.5 \%$ ).

Page 3/10 
A comparison between demographic characteristics and comorbidities between COVID-19 positive and negative tested patients showed no significant differences between the groups (Table 1).

Similar numbers of outpatients tested positive (11.1\%) or negative (8.6\%; $\mathrm{p}=0.338)$. There was also no significant difference between COVID-19 positive compared to negative tested patients regarding the number of patients requiring ICU and/or ventilation (4.0\% vs. $9.3 \%$, respectively; $p=0.091)$. Significantly more COVID-19 positive (25.5\%) than negative (9.2\%) tested patients died before day 28 ( $p<0.001)$. Main causes of death in COVID-19-positive patients were, in descending order, pneumonia (67.3\%), followed by multi-organ failure (24.5\%), acute cardiac failure (7.6\%), and acute renal failure $(1.9 \%)$.

\section{Comparison of Standard Blood Laboratory Parameters between Covid-19 Positive and Negative Patients}

COVID-19 positive patients had significantly lower levels of leukocytes, neutrophils, basophils, eosinophils, lymphocytes, NLR, monocytes, and thrombocytes; while significantly higher levels were detected with erythrocytes, hemoglobin, hematocrit, CRP, ferritin, aPTT, ALT, AST, lipase, CK, and LDH compared to COVID-19 negative patients. Similar levels were detected regarding procalcitonin, albumin, glucose, potassium, total bilirubin, GGT, creatinine, and BUN between the groups (Supplement Table 1).

From all evaluated continuous blood parameters, eosinophils (79.4\%), ferritin (76.4\%), leukocytes (72.3\%), and erythrocytes (72.3\%) showed the highest AUC regarding their ability to distinguish between COVID-19 positive and negative patients (Table 2).

Accuracy, sensitivity, specificity, PPV, NPV, LR+, LR-, as well as pre-test and post-test probabilities are presented in Table 3. Considering LR+ greater than one (e.g. 10 very strong) and LR- less than one (e.g. 0.1 very strong) are progressively stronger, leukopenia, eosinopenia, as well as elevated erythrocytes and hemoglobin were among the strongest regarding meaningful differences/changes from the pre-test probability.

\section{Discussion}

In this trial, we showed that the likelihood of a SARS-CoV-2 infection can be enforced through standard laboratory blood findings to a high degree. Several studies including meta-analyses recently focused on prediction of the severity of the disease derived from blood results. ${ }^{8,9,16}$ Our consideration to find a certain blood pattern to diagnose SARS-CoV-2 infection with standard blood parameters has been less studied.

To our knowledge, only three other trials comparing standard blood parameters between positive and negative cases are published to date. ${ }^{11,12,17}$ Similar to those studies, our study showed that leukopenia, eosinopenia, elevated erythrocytes and hemoglobin, and ferritin were detected to be among the best standard laboratory parameters to distinguish between COVID-19 positive from negative tested patients. Accordingly, similar patterns have been detected in positive COVID-19 patients with a severe compared to a mild form of the disease. 8,9,16 The major differences of the three studies, which compared COVID-19 positive and negative patients, opposed to the reviews, which reported only on positive tested patients, were documented regarding leucocytes, neutrophils, and hemoglobin (Table 4).

In conformity with other publications $11,12,17$, leucocytes were lower in COVID-19 positive than negative patients at the time of PCR testing, and so were neutrophils and lymphocytes; while severe compared to mild forms of COVID-19 tend to have higher leucocytes and neutrophils. As opposed to our findings, which showed a weak ability of NLR to discriminate between positive and negative (AUC=0.443); a raised NLR, which evolved from a raised neutrophil count as well as a lowered lymphocyte count, was already shown previously to be a prognostic value for endotracheal intubation and mortality predictor. ${ }^{13,14}$ A cut-off of 4.94 was used in the publication by Tatum et al. ${ }^{14}$; above this value, the risk of being artificially ventilated or to die was increased. Notably, $89 \%$ of those patients were African Americans. A lower cut-off (2.33) was established in our study, which might be because only $15 \%$ of patients had a neutrophil count higher than $7.7 \times 10^{9} / \mathrm{L}$. We are however unaware of any study using NLR as a pure discriminator between positive and negative COVID-19 diagnosis.

However, severity of illness appears to be less important regarding the other parameters, especially regarding eosinophils and CRP (Table 4). Like in other publications ${ }^{12,17}$, our data also showed that eosinopenia was one of the significant predictive biomarkers for COVID-19 with a sensitivity of $47 \%$ and a specificity of $87 \%$.

Li et al. ${ }^{12}$ and our study showed an increased hemoglobin in COVID-19 positive patients, which is not in accordance with a lowered hemoglobin in patients with severe COVID-19 disease reported by two meta-analyses. ${ }^{8,16}$ In our data, the median hemoglobin was $13.5 \mathrm{~g} / \mathrm{dL}$, which did not much differ from Li's data. ${ }^{12}$ In several other trials assessing the severity of disease and blood patterns, hemoglobin was shown to be below normal ranges. ${ }^{8,9,16}$ It can only be hypothesized why our cohort presented with a comparably high level of hemoglobin. Possibly, a degree of dehydration played a role at the time of presentation in the emergency department. Indeed, an average temperature of $38.0 \pm 0.9^{\circ} \mathrm{C}$ on presentation in $99 \mathrm{COVID-}$ 19 positive and $37.1 \pm 1.4^{\circ} \mathrm{C}$ in 103 COVID-19 negative patients, which was a significant difference, was detected in a subgroup analysis of 202 of our patients.

Not surprisingly, CRP was significantly elevated in all studies. ${ }^{11,12,17}$ In our patients, we set a new cut-off at $22 \mathrm{mg} / \mathrm{dL}$, since the vast majority of patients had increased CRP values. 
Brinati et al. included 279 patients and developed a score for SARS-Cov- 2 detection with an accuracy between $82 \%$ and $86 \%$, and sensitivity between $92 \%$ and $95 \% .{ }^{18}$ Applying our data including age, gender, leucocytes, neutrophils, lymphocytes, monocytes, eosinophils, basophils, thrombocytes, CRP, AST, ALT, GGT, and LDH to Brinati's tool, a quite high AUC (AUC=0.709, 95\% Cl 0.646-0.771; $p<0.001$ ), sensitivity (70.4\%), specificity (71.3\%), and NPV (79.9\%), but less promising PPV (59.8\%) could be obtained. However, our model including 14 standard laboratory blood parameters reached better diagnostic performances in all areas (AUC=0.915, 95\% Cl 0.876-0.955); sensitivity (78.4\%); specificity (87.3\%), PPV (79.5\%), and NNP (86.6\%)), although, the most prominent parameters were leucocytes, eosinophils, hemoglobin, and CRP.

The following limitations of the study should be noted. The retrospective design with missing blood parameters are amongst the major limiting factors. Additionally, with the single time point evaluation, we were not able to retrieve information regarding progression of the disease. Furthermore, cytokines, especially interleukin-6, were not routinely measured, which may be better predictors, especially regarding the so-called ,COVID-19 cytokine storm', to elucidate COVID-19 positive from negative patients. Another fact to consider is the heterogeneity of underlying diseases, which may also contribute to variations in our findings. On the other hand, such a heterogeneity may reflect reality during a pandemic situation best. Eventually, all test quality crucially depends on the quality of the manual specimen acquisition. ${ }^{19,20}$ PCR results tend to be more positive in patients with an increased viral load and with a shorter duration of the disease. ${ }^{21}$

Generally, as laboratory equipment supply develops, more PCR point-of-care diagnostics become available. It is nonetheless doubtful that - neither in the near, nor in the far future - PCR will entirely replace standard laboratory testing. Therefore, the question of a blood laboratory pattern, as specific as possible for COVID-19, remains relevant. Our investigation showed that especially leukopenia, eosinopenia, as well as elevated erythrocytes, hemoglobin, and ferritin are among the best markers to distinguish between COVID-19 positive and negative patients. Therefore, such biomarkers could be useful to facilitate rapid triage of potential COVID-19 patients.

\section{Declarations}

\section{Author contributions}

R.T. and B.L. designed the study, took responsibility for integrity and accuracy of data, and drafted the manuscript. R.T. and J.Z. had full access to all data in the study and contributed to collection of data. B.L. performed statistical analysis. R.T. and B.L. made critical revisions to this manuscript. All authors contributed to interpretation of data, read, reviewed, and approved the final version of the manuscript.

\section{Competing interests}

The authors declare no competing interests.

\section{Funding}

There was no acquisition of any funding for this trial.

\section{Additional information}

The datasets generated and/or analysed during the current study are available from the corresponding author on reasonable request.

\section{References}

1. Wang, L. F., Anderson, D. E., Mackenzie, J. S. \& Merson, M. H. From Hendra to Wuhan: what has been learned in responding to emerging zoonotic viruses. Lancet395, e33-e34, doi:10.1016/S0140-6736(20)30350-0 (2020).

2. Wang, Y., Kang, H., Liu, X. \& Tong, Z. Combination of RT-qPCR testing and clinical features for diagnosis of COVID-19 facilitates management of SARS-CoV-2 outbreak. J Med Viro/92, 538-539, doi:10.1002/jmv.25721 (2020).

3. Chen, N. et al. Epidemiological and clinical characteristics of 99 cases of 2019 novel coronavirus pneumonia in Wuhan, China: a descriptive study. Lancet395, 507-513, doi:10.1016/S0140-6736(20)30211-7 (2020).

4. Guan, W. J. et al. Clinical Characteristics of Coronavirus Disease 2019 in China. N Engl J Med382, 1708-1720, doi:10.1056/NEJMoa2002032 (2020).

5. Gandhi, R. T., Lynch, J. B. \& Del Rio, C. Mild or Moderate Covid-19. N Engl J Med, doi:10.1056/NEJMcp2009249 (2020).

6. Mattiuzzi, C. \& Lippi, G. Which lessons shall we learn from the 2019 novel coronavirus outbreak? Ann Trans/ Med8, 48, doi:10.21037/atm.2020.02.06 (2020).

7. Zhang, G. et al. Analysis of clinical characteristics and laboratory findings of 95 cases of 2019 novel coronavirus pneumonia in Wuhan, China: a retrospective analysis. Respir Res21, 74, doi:10.1186/s12931-020-01338-8 (2020). 
8. Henry, B. M., de Oliveira, M. H. S., Benoit, S., Plebani, M. \& Lippi, G. Hematologic, biochemical and immune biomarker abnormalities associated with severe illness and mortality in coronavirus disease 2019 (COVID-19): a meta-analysis. Clin Chem Lab Med58, 1021-1028, doi:10.1515/cclm-2020-0369 (2020).

9. Ponti, G., Maccaferri, M., Ruini, C., Tomasi, A. \& Ozben, T. Biomarkers associated with COVID-19 disease progression. Crit Rev Clin Lab Sci, 1-11, doi:10.1080/10408363.2020.1770685 (2020).

10. Wang, D. et al. Clinical Characteristics of 138 Hospitalized Patients With 2019 Novel Coronavirus-Infected Pneumonia in Wuhan, China. JAMA, doi:10.1001/jama.2020.1585 (2020).

11. Mardani, R. et al. Laboratory Parameters in Detection of COVID-19 Patients with Positive RT-PCR; a Diagnostic Accuracy Study. Arch Acad Emerg Med8, e43 (2020).

12. Li, Q. et al. Eosinopenia and elevated C-reactive protein facilitate triage of COVID-19 patients in fever clinic: a retrospective case-control study. EClinicalMedicine, 100375, doi:10.1016/j.eclinm.2020.100375 (2020).

13. Liu, Y. et al. Neutrophil-to-lymphocyte ratio as an independent risk factor for mortality in hospitalized patients with COVID-19. J Infect81, e6e12, doi:10.1016/j.jinf.2020.04.002 (2020).

14. Tatum, D. et al. Neutrophil-to-Lymphocyte Ratio and Outcomes in Louisiana Covid-19 Patients. Shock, doi:10.1097/SHK.0000000000001585 (2020).

15. Lippi, G. \& Plebani, M. Laboratory abnormalities in patients with COVID-2019 infection. Clin Chem Lab Med58, 1131-1134, doi:10.1515/cclm2020-0198 (2020).

16. Alnor, A., Sandberg, M. B., Gils, C. \& Vinholt, P. J. Laboratory tests and outcome for patients with COVID-19: A systematic review and metaanalysis. J App/ Lab Med, doi:10.1093/jalm/jfaa098 (2020).

17. Ferrari, D., Motta, A., Strollo, M., Banfi, G. \& Locatelli, M. Routine blood tests as a potential diagnostic tool for COVID-19. Clin Chem Lab Med58, 1095-1099, doi:10.1515/cclm-2020-0398 (2020).

18. Brinati, D. et al. Detection of COVID-19 Infection from Routine Blood Exams with Machine Learning: A Feasibility Study. J Med Syst44, 135, doi:10.1007/s10916-020-01597-4 (2020).

19. Tu, Y. P. et al. Swabs Collected by Patients or Health Care Workers for SARS-CoV-2 Testing. N Engl J Med383, 494-496, doi:10.1056/NEJMc2016321 (2020).

20. Wang, W. et al. Detection of SARS-CoV-2 in Different Types of Clinical Specimens. JAMA, doi:10.1001/jama.2020.3786 (2020).

21. Gorzer, I. et al. First results of a national external quality assessment scheme for the detection of SARS-CoV-2 genome sequences. $J$ Clin Viro/129, 104537, doi:10.1016/j.jcv.2020.104537 (2020).

\section{Tables}

Table 1. Comparison of Demographic Characteristics and 28-day Clinical Outcome between COVID-19 Positive and Negative tested Patients. 


\begin{tabular}{|c|c|c|c|}
\hline \multirow[t]{3}{*}{ Characteristic } & COVID-19 & COVID-19 & \multirow[t]{3}{*}{$P$ Value } \\
\hline & Positive & Negative & \\
\hline & $(\mathrm{N}=208)$ & $(\mathrm{N}=382)$ & \\
\hline Male - no./total no. (\%) & $117(56.3)$ & $197(51.6)$ & $0.276^{*}$ \\
\hline \multicolumn{4}{|l|}{ Age } \\
\hline Median (IQR) - yr & $72(57-79.75)$ & $70(54-81)$ & $0.526^{+}$ \\
\hline \multicolumn{4}{|l|}{ Comorbidities - no./total no. (\%) } \\
\hline Hypertension & $114(54.8)$ & $229(59.9)$ & $0.227 *$ \\
\hline Diabetes & $50(24.0)$ & $100(26.2)$ & $0.569 *$ \\
\hline Coronary heart disease* & 33 (15.9) & $82(21.5)$ & $0.101^{*}$ \\
\hline Chronic lung disease $^{\dagger}$ & $22(10.6)$ & $78(20.4)$ & $0.002^{*}$ \\
\hline Chronic kidney disease ${ }^{\ddagger}$ & $29(13.9)$ & $64(16.8)$ & $0.371 *$ \\
\hline Malignant tumor $\S$ & $12(5.8)$ & $68(17.8)$ & $<0.001^{*}$ \\
\hline Cerebro vascular accident $₫$ & $13(6.3)$ & $31(8.1)$ & $0.410 *$ \\
\hline Chronic liver diseasell & $2(1.0)$ & $23(6.0)$ & $0.004^{*}$ \\
\hline Human immunodeficiency virus & 0 & $3(0.8)$ & $0.556^{\ddagger}$ \\
\hline \multicolumn{4}{|l|}{ Clinical 28-day outcome - no. (\%) } \\
\hline Not hospitalized/outpatient & $23(11.1)$ & $33(8.6)$ & \multirow[t]{5}{*}{$<0.001^{*}$} \\
\hline $\begin{array}{l}\text { Discharged after } \leq 28 \text { days hospitalization } \\
\text { not requiring ICU support or ventilation }\end{array}$ & $97(46.6)$ & $245(64.1)$ & \\
\hline $\begin{array}{l}\text { Discharged after } \leq 28 \text { days hospitalization } \\
\text { requiring ICU support and/or ventilation }\end{array}$ & $4(1.9)$ & $25(6.5)$ & \\
\hline still in hospital after day 28 & $31(14.9)$ & $44(11.5)$ & \\
\hline Died before day 28 & $53(25.5)$ & $35(9.2)$ & \\
\hline
\end{tabular}

Abbreviation: COVID-19, coronavirus disease 2019; ICU, intensive care unit; IQR, interquartile range

* Chi-square test; ${ }^{\dagger}$ Mann-Whitney U-test; ${ }^{\ddagger}$ Fischer's exact test

Table 2. Diagnostic Performance of Each Continuous Blood Laboratory Parameters to Distinguish Between COVID-19 Positive from Negative Tested Patients. 


\begin{tabular}{|c|c|c|}
\hline Parameters & $\operatorname{AUC}(95 \% \mathrm{Cl})$ & P-value \\
\hline Leukocytes $\left(10^{9} / \mathrm{L}\right)$ & $\begin{array}{l}0.277 \\
(0.235-0.320)\end{array}$ & $<0.001$ \\
\hline Neutrophils $\left(10^{9} / \mathrm{L}\right)$ & $\begin{array}{l}0.299 \\
(0.252-0.346)\end{array}$ & $<0.001$ \\
\hline Basophils $\left(10^{9} / \mathrm{L}\right)$ & $\begin{array}{l}0.300 \\
(0.253-0.346)\end{array}$ & $<0.001$ \\
\hline Eosinophils $\left(10^{9} / \mathrm{L}\right)$ & $\begin{array}{l}0.206 \\
(0.164-0.249)\end{array}$ & $<0.001$ \\
\hline Lymphocytes $\left(10^{9} / \mathrm{L}\right)$ & $\begin{array}{l}0.376 \\
(0.327-0.425)\end{array}$ & $<0.001$ \\
\hline Neutrophil-to lymphocyte rate & $\begin{array}{l}0.443 \\
(0.391-0.496)\end{array}$ & 0.032 \\
\hline Monocytes (10\%/L) & $\begin{array}{l}0.394 \\
(0.344-0.445)\end{array}$ & $<0.001$ \\
\hline Thrombocytes $\left(10^{9} / \mathrm{L}\right)$ & $\begin{array}{l}0.423 \\
(0.376-0.470)\end{array}$ & 0.002 \\
\hline Erythrocytes $\left(10^{12} / \mathrm{L}\right)$ & $\begin{array}{l}0.723 \\
(0.681-0.765)\end{array}$ & $<0.001$ \\
\hline Hemoglobin (g/dL) & $\begin{array}{l}0.697 \\
(0.653-0.740)\end{array}$ & $<0.001$ \\
\hline Hematocrit (\%) & $\begin{array}{l}0.668 \\
(0.623-0.712)\end{array}$ & $<0.001$ \\
\hline $\mathrm{CRP}(\mathrm{mg} / \mathrm{dL})$ & $\begin{array}{l}0.610 \\
(0.0563-0.657)\end{array}$ & $<0.001$ \\
\hline Ferritin $(\mathrm{mcg} / \mathrm{L})$ & $\begin{array}{l}0.764 \\
(0.660-0.868)\end{array}$ & $<0.001$ \\
\hline aPTT (sec) & $\begin{array}{l}0.619 \\
(0.564-0.674)\end{array}$ & $<0.001$ \\
\hline $\operatorname{ALT}(\mathrm{U} / \mathrm{L})$ & $\begin{array}{l}0.592 \\
(0.541-0.642)\end{array}$ & 0.001 \\
\hline AST (U/L) & $\begin{array}{l}0.657 \\
(0.597-0.716)\end{array}$ & $<0.001$ \\
\hline Lipase (U/L) & $\begin{array}{l}0.684 \\
(0.632-0.735)\end{array}$ & $<0.001$ \\
\hline CK (U/L) & $\begin{array}{l}0.561 \\
(0.510-0.611)\end{array}$ & 0.023 \\
\hline LDH (U/L) & $\begin{array}{l}0.659 \\
(0.607-0.711)\end{array}$ & $<0.001$ \\
\hline
\end{tabular}


Abbreviations: ALT, alanine aminotransferase; aPTT, activated partial thromboplastin time; AST, aspartate aminotransferase; AUC, area under the curve; CK, creatine kinase; CRP, C-reactive protein; LDH, lactate dehydrogenase; NLR, Neutrophil-to-lymphocyte ratio.

Table 3. Diagnostic Performance of Single Standard Categorical Blood Laboratory Parameters to Distinguish Between COVID-19 Positive from Negative Tested Patients.

\begin{tabular}{|c|c|c|c|c|c|c|c|c|c|c|c|}
\hline Parameters & Accuracy & Sensitivity & Specificity & PPV & NPV & LR+ & LR- & $\begin{array}{l}\text { pre-test } \\
\text { probability }\end{array}$ & $\begin{array}{l}\text { post-test } \\
\text { (pos) } \\
\text { probability }\end{array}$ & $\begin{array}{l}\text { post-test } \\
\text { (neg) } \\
\text { probability }\end{array}$ & $\begin{array}{l}\text { change in } \\
\text { (pos/neg) } \\
\text { probability } \\
(\%)\end{array}$ \\
\hline $\begin{array}{l}\text { leucocytes } \\
\left(<=1010^{9} / \mathrm{L}\right)\end{array}$ & 0.574 & 0.448 & 0.849 & 0.865 & 0.415 & 2.96 & 0.65 & 0.684 & 0.865 & 0.585 & $18.1 /-9.9$ \\
\hline $\begin{array}{l}\text { neutrophils } \\
(<0.10 \\
\left.10^{9} / \mathrm{L}\right)\end{array}$ & 0.543 & 0.416 & 0.829 & 0.846 & 0.386 & 2.44 & 0.70 & 0.693 & 0.846 & 0.614 & $15.3 /-7.9$ \\
\hline $\begin{array}{l}\text { eosinophils } \\
(<0.10 \\
\left.10^{9} / \mathrm{L}\right)\end{array}$ & 0.620 & 0.468 & 0.867 & 0.852 & 0.500 & 3.52 & 0.61 & 0.620 & 0.852 & 0.500 & $\begin{array}{l}23.2 / \\
-12.0\end{array}$ \\
\hline $\begin{array}{l}\text { lymphocytes } \\
\left(<1.510^{9} / \mathrm{L}\right)\end{array}$ & 0.506 & 0.393 & 0.789 & 0.824 & 0.341 & 1.87 & 0.77 & 0.715 & 0.824 & 0.659 & $10.9 /-5.6$ \\
\hline $\operatorname{NLR}(\leq 2.33)$ & 0.324 & 0.310 & 0.429 & 0.802 & 0.077 & 0.54 & 1.61 & 0.882 & 0.802 & 0.923 & $-8.0 / 4.1$ \\
\hline $\begin{array}{l}\text { monocytes } \\
\left(\leq 0.910^{9} / \mathrm{L}\right)\end{array}$ & 0.416 & 0.361 & 0.803 & 0.929 & 0.151 & 1.83 & 0.80 & 0.876 & 0.929 & 0.849 & $5.2 /-2.7$ \\
\hline $\begin{array}{l}\text { erythrocytes } \\
(\geq 4.3 \\
\left.10^{12} / \mathrm{L}\right)\end{array}$ & 0.655 & 0.509 & 0.784 & 0.676 & 0.643 & 2.36 & 0.63 & 0.470 & 0.676 & 0.357 & $\begin{array}{l}20.6 / \\
-11.3\end{array}$ \\
\hline $\begin{array}{l}\text { hemoglobin } \\
\text { (f: } \geq 11.8 ; \mathrm{m}: \\
\geq 13.5 \mathrm{~g} / \mathrm{dL})\end{array}$ & 0.639 & 0.493 & 0.777 & 0.676 & 0.619 & 2.21 & 0.65 & 0.485 & 0.676 & 0.381 & $\begin{array}{l}19.1 / \\
-10.5\end{array}$ \\
\hline $\begin{array}{l}\text { hematocrit } \\
\text { (f: >38.0\%; } \\
\text { m: } 39.5 \%)\end{array}$ & 0.651 & 0.506 & 0.746 & 0.565 & 0.698 & 1.99 & 0.66 & 0.395 & 0.565 & 0.302 & $17.0 /-9.3$ \\
\hline $\begin{array}{l}\mathrm{CRP}(\geq 22 \\
\mathrm{mg} / \mathrm{dL})\end{array}$ & 0.549 & 0.423 & 0.785 & 0.785 & 0.423 & 1.97 & 0.74 & 0.650 & 0.785 & 0.577 & $13.5 /-7.3$ \\
\hline $\begin{array}{l}\text { procalcitonin } \\
(\leq 0.5 \\
\mathrm{ng} / \mathrm{mL})\end{array}$ & 0.485 & 0.382 & 0.857 & 0.907 & 0.276 & 2.68 & 0.72 & 0.785 & 0.907 & 0.724 & $12.2 /-6.0$ \\
\hline $\begin{array}{l}\text { aPTT (>32 } \\
\text { sec) }\end{array}$ & 0.663 & 0.469 & 0.730 & 0.376 & 0.799 & 1.74 & 0.73 & 0.257 & 0.376 & 0.201 & $11.8 /-5.6$ \\
\hline $\begin{array}{l}\mathrm{ALT}(>45 \\
\mathrm{U} / \mathrm{L})\end{array}$ & 0.621 & 0.414 & 0.702 & 0.351 & 0.754 & 1.39 & 0.84 & 0.280 & 0.351 & 0.246 & $7.0 /-3.5$ \\
\hline $\begin{array}{l}\text { AST (>35 } \\
\text { U/L) }\end{array}$ & 0.632 & 0.510 & 0.739 & 0.629 & 0.634 & 1.95 & 0.66 & 0.465 & 0.629 & 0.366 & $16.4 /-9.9$ \\
\hline $\begin{array}{l}\text { bilirubin total } \\
(\leq 1.0 \mathrm{mg} / \mathrm{dL})\end{array}$ & 0.419 & 0.355 & 0.800 & 0.913 & 0.173 & 1.78 & 0.81 & 0.856 & 0.913 & 0.827 & $5.8 /-2.9$ \\
\hline $\begin{array}{l}\text { lipase }(>60 \\
\mathrm{U} / \mathrm{L})\end{array}$ & 0.658 & 0.517 & 0.694 & 0.298 & 0.851 & 1.69 & 0.70 & 0.201 & 0.298 & 0.149 & $9.7 /-5.2$ \\
\hline $\begin{array}{l}\text { CK (>190 } \\
\text { U/L) }\end{array}$ & 0.607 & 0.430 & 0.685 & 0.376 & 0.732 & 1.37 & 0.83 & 0.306 & 0.376 & 0.268 & $7.0 /-3.8$ \\
\hline $\begin{array}{l}\mathrm{LDH}(>250 \\
\mathrm{U} / \mathrm{L})\end{array}$ & 0.610 & 0.457 & 0.759 & 0.650 & 0.588 & 1.90 & 0.72 & 0.494 & 0.650 & 0.412 & $15.5 /-8.3$ \\
\hline $\begin{array}{l}\text { creatinine } \\
(\geq 0.5 \\
\mathrm{mg} / \mathrm{dL})\end{array}$ & 0.376 & 0.363 & 1.000 & 1.000 & 0.032 & - & 0.64 & 0.979 & - & 0.968 & $-/-1.1$ \\
\hline
\end{tabular}


Abbreviations: ALT, alanine aminotransferase; aPTT, activated partial thromboplastin time; AST, aspartate aminotransferase; AUC, area under the curve; CK, creatine kinase; CRP, C-reactive protein; LDH, lactate dehydrogenase; NLR, Neutrophil-to-lymphocyte ratio.

Table 4. Blood parameter patterns of COVID-19 positive tested patients of various studies.

\begin{tabular}{|llll|}
\hline & Present study & COVID-19 & positive COVID-19 \\
& COVID-19 positive & positive & severe \\
\hline Leucocytes & $\downarrow$ & $\downarrow^{11,12,17}$ & $\uparrow^{8,9,16}$ \\
\hline Neutrophils & $\downarrow$ & $\downarrow^{11,12,17}$ & $\uparrow^{8,9,16}$ \\
\hline Lymphocytes & $\downarrow$ & $\downarrow^{11,12,17}$ & $\downarrow^{8,9,16}$ \\
\hline Thrombocytes & $\downarrow$ & $\downarrow^{12,17}$ & $\downarrow^{8,9}$ \\
\hline Hemoglobin & $\uparrow$ & $\downarrow^{12} ;$ not sig. $\downarrow^{17}$ & $\downarrow^{8,9,16}$ \\
\hline CRP & $\uparrow$ & not sig. $\uparrow^{12}$ & $\downarrow^{8,16}$ \\
\hline ALT & $\uparrow$ & $\uparrow^{11,12,17}$ & $\uparrow^{8,9,16}$ \\
\hline AST & $\uparrow$ & $\uparrow^{12,17}$ & $\uparrow^{8,9}$ \\
\hline LDH & $\uparrow$ & $\uparrow^{12,17}$ & $\uparrow^{8,9,16}$ \\
\hline
\end{tabular}

Abbreviations: ALT, alanine aminotransferase; AST, aspartate aminotransferase; CRP, C-reactive protein; LDH, lactate dehydrogenase.

\section{Supplementary Files}

This is a list of supplementary files associated with this preprint. Click to download.

- Supplementfile.pdf 